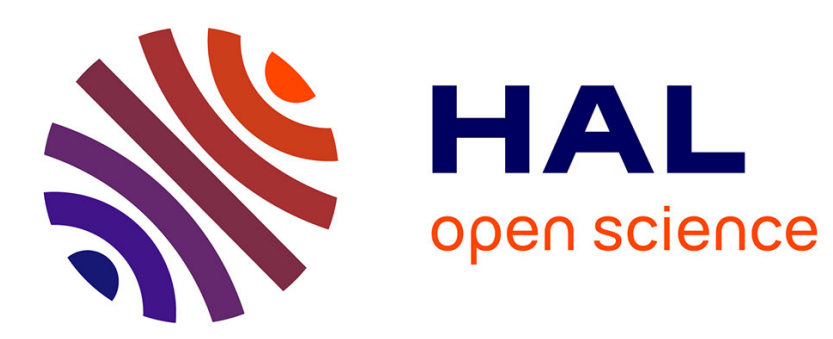

\title{
Effet d'une perturbation sur l'estimation de modèles autorégressifs
}

\author{
T. Robert, Corinne Mailhes
}

\section{To cite this version:}

T. Robert, Corinne Mailhes. Effet d'une perturbation sur l'estimation de modèles autorégressifs. Journal de Physique IV Proceedings, 1994, 04 (C5), pp.C5-1383-C5-1386. 10.1051/jp4:19945308 . jpa-00253023

\section{HAL Id: jpa-00253023 https://hal.science/jpa-00253023}

Submitted on 1 Jan 1994

HAL is a multi-disciplinary open access archive for the deposit and dissemination of scientific research documents, whether they are published or not. The documents may come from teaching and research institutions in France or abroad, or from public or private research centers.
L'archive ouverte pluridisciplinaire HAL, est destinée au dépôt et à la diffusion de documents scientifiques de niveau recherche, publiés ou non, émanant des établissements d'enseignement et de recherche français ou étrangers, des laboratoires publics ou privés. 


\title{
Effet d'une perturbation sur l'estimation de modèles autorégressifs
}

\author{
T. ROBERT et C. MAILHES
}

ENSEEIHT/GAPSE, 2 nue Camichel, 31071 Toulouse, France

In spectral analysis and pattern recognition, especially in speech processing, AutoRegressive (AR) modeling is widely used as a signal parameter estimation tonl. In this paper, AR estimation behaviour is studied when applied to signals presenting abrupt changes at an unknown instant of time. A particular abrupt change is studied : the additive change case. This change occurs when, at the instant of change, a signal adds to one which was present before - both signals are independent -. The theoretical sliding window AR parameter expressions are given. We show that blind sliding window AR estimation can lead to a detection tool, allowing additive changes to be detected and distinguished.

\section{Introduction}

En traitement de la parole, la difficulté principale réside dans le caractère non-stationnaire des signaux à analyser. Toutefois, cette difficulté peut être levée en considérant le signal stationnaire par morceaux, même s'il faut prendre en compte des ruptures brutales qui apparaissent à des instants inconnus. Ces ruptures peuvent être de natures différentes. Dans cet article, nous étudions un cas particulier de rupture : le cas de rupture additive. Ce type de rupture se caractérise par l'addition à un instant donné, d'un signal indépendant du premier. En pratique; cela correspond à l'analyse d'un signal de parole perturbé à un instant donné, par l'addition d'un second signal parole (autre locuteur) ou d'un autre son.

En traitement de la parole, un des modèles paramétriques couramment employés est le modèle AutoRégressif(AR) [1]. Dans le cas d'une modélisation AR glissante, la détermination des paramètres du modèle se fait sur une fenêtre de taille constante qui se déplace sur le signal. Si le signal est stationnaire, quelque soit l'instant du début de la fenêtre, les paramètres AR sont identiques. Dans le cas qui nous intéresse, le signal est non-stationnaire. A un instant donné, un second signal (signal perturbateur) indépendant du premier vient se rajouter à celui-ci.L'estimation AR glissante et aveugle, c'est-à-dire sans detection préalable de l'instant de la rupture, conduit au même système d'équations que dans le cas stationnaire, mais, dans ce cas, les paramètres AR dépendent de la position de la fenêtre de modélisation.

Tout d'abord, nous donnons l'expression théorique des paramètres AR issus de la modélisation glissante le long de la rupture. Ensuite, nous présentons une application de cette étude théorique conduisant à une détection de la rupture. 


\section{Analyse du Signal}

Le signal $y(n)$ que l'on cherche à analyser s'écrit :

$y(n)=\mid \begin{aligned} & x_{1}(n) \quad \text { si } n \leq N_{r} \\ & x_{2}(n)=x_{1}(n)+x_{3}(n) \quad \text { si } n>N_{r}\end{aligned}$

$N_{r}$ étant l'instant de rupture,

avec $x_{1}(n)=-\sum_{k=1}^{p_{1}} a(k) x_{1}(n-k)+e_{1}(n)$ un signal AR d'ordre $p_{1}$ et de vecteur paramètre $\underline{a}$, et $\quad x_{3}(n)=-\sum_{k=1}^{p_{3}} b(k) x_{3}(n-k)+e_{3}(n)$ un signal AR d'ordre $p_{3}$ de vecteur paramètre $\underline{b}$,

$x_{1}(n)$ et $x_{3}(n)$ étant indépendants.

Dans le cas d'une modélisation AR glissante d'ordre $L$ sur la fenêtre $[(n-L), \ldots, n], y(n)$ est représenté par :

$$
y(n)=-\sum_{k=1}^{L} c_{n}(k) y(n-k)+e(n)
$$

$e(n)$ étant un bruit blanc gaussien et $\underline{c}_{n}$ le vecteur paramètre à un instant $n$.

L'estimation des paramètres du modèle $\mathrm{AR}$ conduit aux équations de Yule \& Walker [1] :

$$
\underline{c}_{n}=-R_{y_{n}}^{-1} r_{y_{n}}
$$

où $R_{y_{n}}$ est la matrice de corrélation dont les éléments s'écrivent :

$E\{y(n-i) y(n-j)\}=r_{y_{n}}(n-i, n-j)$ pour $i, j=1, \ldots, L$

et $r_{y_{n}}$ est le vecteur de corrélation de composantes : $r_{y_{n}}(n, n-j)$ pour $j=1, \ldots, L$

Du fait de la non stationnarité, la valeur de la fonction d'autocorrélation $r_{y_{n}}(n-i, n-j)$ dépend de l'instant $n$ considéré. Il y a trois cas à étudier :

* si $n \leq N_{r}$ alors

$\mid \begin{aligned} & R_{y_{n}}=R_{x_{1}} \\ & r_{y_{n}}=\underline{r}_{x_{1}}\end{aligned} \quad$ donc $\underline{c}_{n}=\underline{a}$ vecteur paramètre AR de la modélisation du premier signal $x_{1}(n)$.

* si $\quad n \geq N_{r}+L+1$ alors

$\mid \begin{aligned} & R_{y_{n}}=R_{x_{2}} \\ & r_{y_{n}}=\underline{r}_{x_{2}}\end{aligned}$ donc $\underline{c}_{n}=\underline{c}=-\left(R_{x_{1}}+R_{x_{3}}\right)^{-1} \cdot\left(\underline{r}_{x_{1}}+\underline{r}_{x_{3}}\right)$ vecteur paramètre AR de la modélisation

du second signal $x_{2}(n)$.

* si $N_{r}+1 \leq n \leq N_{r}+L$ alors :

$$
R_{y_{n}}=R_{x_{1}}+R_{x_{3} n} \quad \text { et } \quad \underline{r}_{-y_{n}}^{t}=\left(\underline{r}_{x_{1}}+\underline{r}_{x_{3} n}\right)^{t}
$$

avec

$\underline{r}_{x_{1}}^{2}=\left[\begin{array}{llll}r_{x_{1}}(1) & \ldots & r_{x_{1}}(L)\end{array}\right], \underline{r}_{x_{3} n}^{t}=\left[\begin{array}{lllllll}r_{x_{3}}(1) & \ldots & r_{x_{3}}(n-N r-1) & 0 & \ldots & 0\end{array}\right]$

et

$R_{x_{1}}=\left(\begin{array}{ccc}r_{x_{1}}(0) & \ldots & r_{x_{1}}(L-1) \\ \vdots & & \vdots \\ r_{x_{1}}(L-1) & \ldots & r_{x_{3}}(0)\end{array}\right) \cdot R_{x_{2 x}}=\left(\begin{array}{cccccc}r_{* 3}(0) & \ldots & r_{x_{3}}(n-N r-2) & 0 & \ldots & 0 \\ \vdots & & \vdots & \vdots & & \vdots \\ r_{x_{3}(n-N r-2)} & \ldots & r_{x_{3}}(0) & 0 & \ldots & 0 \\ 0 & \ldots & 0 & \ldots & 0 \\ \vdots & & \vdots & \vdots & & \vdots \\ 0 & \ldots & 0 & 0 & \ldots & 0\end{array}\right)$ 
A partir du lemme d'inversion, on peut déterminer la matrice $R_{y_{k}}^{-1}$ :

$R_{y_{n}}^{-1}=\left(R_{x_{1}}+R_{x_{3^{n}}}\right)^{-1}=R_{x_{1}}^{-1}-R_{x_{1}}^{-1} \cdot R_{x_{3} n} \cdot\left(R_{x_{1}}^{-1} \cdot R_{x_{3} n}+I^{-1}\right)^{-1} \cdot R_{x_{1}}^{-1}=R_{x_{1}}^{-1}+\delta\left(R_{x_{1}}^{-1}\right)(n)$

On voit donc que la perturbation apportée par le signal $x_{3}(n)$ sur le premier modèle c'est-à-dire $x_{1}(n)$ induit une perturbation $\delta\left(R_{x_{1}}^{-1}\right)(n)$ sur $R_{x_{1}}^{-1}$.

On peut alors calculer la valeur de $\underline{c}_{n}$ et on trouve :

$\underline{c}_{n}=-\left(R_{x_{1}}+R_{x_{3^{n}}}\right)^{-1}\left(r_{x_{1}}+\underline{r}_{x_{3^{n}}}\right)=-\left(R_{x_{1}}^{-1}+\delta\left(R_{x_{1}}^{-1}\right)(n)\right)\left(\underline{r}_{x_{1}}+\underline{r}_{x_{3^{n}}}\right)=\underline{a}+\delta \underline{a}(n)$

avec $\delta \underline{\alpha}(n)=-R_{x_{1}}^{-1} \cdot \underline{r}_{x_{3}}(n)-\delta\left(R_{x_{1}}^{-1}\right)(n) \cdot \underline{r}_{x_{1}}-\delta\left(R_{x_{1}}^{-1}\right)(n) \cdot \underline{r}_{x_{3}}(n)$

$a$ étant le vecteur des paramètres du premier modèle (dimension $p_{1}$ ) complété par des zéros jusqu'à avoir la dimension $L . \delta \underline{a}(n)$ est la perturbation sur les coefficients en considérant la fenêtre de modélisation $[(n-L), \ldots, n]$.

\section{Exemple}

A titre d'exemple, considérons $N=500$ réalisations d'un processus présentant une rupture additive. L'instant de rupture choisi est $N_{r}=10, x_{1}(n)$ est un modèle AR d'ordre $p_{1}=2$ et de vecteur paramètre $\underline{a}=[1,-0.6,0.8]^{t}, x_{3}(n)$ est un modèle AR d'ordre $p_{3}=2$ et de vecteur paramètre $\underline{b}=[1,0.01,0.4]^{t}$. Dans la mesure où le signal étudié est non stationnaire, il est non ergodique. Pour déterminer les valeurs de $r_{y_{n}}(n-i, n-j)$, nous avons donc réalisé une moyenne statistique sur ces $N=500$ expériences, et ainsi estimé la valeur de $\underline{c}_{n}$ et la puissance du bruit $\sigma_{e}^{2}$.

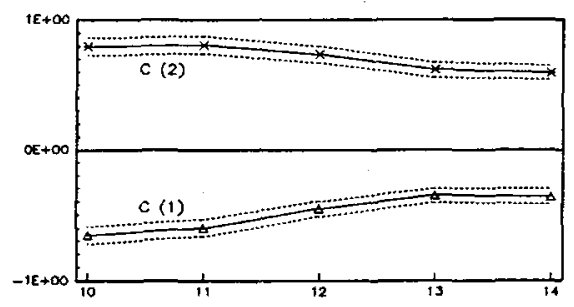

fig 1 : Estimation de $\underline{c}_{n}$ en fonction de $n$ et son

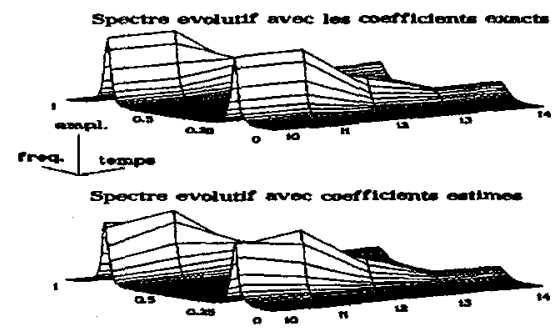

fig 2 : spectre AR évolutif

intervalle de confiance $\grave{a} \pm 2 \sigma_{n}$

La figure 1, présente les résultats de l'estimation des paramètres AR le long de la rupture ainsi que les intervalles de confiance $\mathbf{a} \pm 2 \sigma_{n}$ de part et d'autre de la valeur théorique $\left(\sigma_{n}^{2}=\left(\sigma_{e}^{2} / N\right) \cdot\left(R_{y_{n}}^{-1}\right)\right.$. La figure 2 permet de comparer le spectre $A R$ calculé avec les paramètres $A R$ théoriques (courbe du haut) et celui calculé avec les paramètres estimés (courbe du bas).

Cet exemple permet de confirmer et d'illustrer les résultats théoriques du paragraphe précédent.

\section{Détection en présence de bruit}

Nous utilisons ces résultats théoriques pour construire une méthode de détection de ruptures additives. A l'issue de la modélisation AR glissante effectuée sur le signal $y(n)$, nous disposons d'une matrice dont les lignes corresponcient à l'estimation des paramètres AR à chaque instant $n$ : 


$$
C=\left[\begin{array}{cccccc}
\dot{\hat{c}}_{1}(1) & \dot{\hat{c}}_{1}(2) & \cdot & \cdot & \cdot & \overline{\hat{c}}_{1}(L) \\
\dot{\bar{c}}_{2}(1) & \dot{\bar{c}}_{2}(2) & \cdot & \cdot & \cdot & \dot{\hat{c}}_{2}(L) \\
\cdot & \cdot & & & & \cdot \\
\cdot & \cdot & & & & \cdot \\
\cdot & \cdot & & & & \cdot \\
\dot{\underline{c}}_{n+1}(1) & \hat{\underline{c}}_{n+1}(2) & \cdot & \cdot & \cdot & \hat{\underline{\hat{c}}}_{n+1}(L)
\end{array}\right]
$$

Le problème que l'on se pose ici est celui de la détection dans cette matrice, d'une évolution connue des paramètres AR, donnée par l'expression (2). Cela peut être vu comme un problème de détection d'une forme connue dans une image bruitée. Une des méthodes couramment employée en traitement d'image est basée sur un calcul de corrélation à deux dimensions [2].

Connaissant l'expression théorique des coefficients le long d'une rupture additive (2), nous pouvons déterminer la présence effective de cette rupture et détecter son instant d'apparition, par un calcul de corrélation entre la matrice $\hat{C}$ et la matrice constituée de l'évolution théorique de ces coefficients.

Dans un premier temps, nous déterminons l'expression théorique des coefficients à partir de l'équation (2). Nous appliquons ensuite la méthode de corrélation à un signal présentant une rupture additive à l'instant $N_{r}=12$ (signal généré suivant l'équation (1)). La corrélation obtenue en fonction de l'instant $n$ où s'effectue le calcul est tracée figure 3. Elle présente un "pic" à l'instant de rupture. Dans la figure 4, le même cslcul est effectué sur le même signal bruité. Le "pic" reste visible. Par contre, dans la figure 5 , la même méthode est appliquée à un autre signal présentant une rupture de nature différente au même instant $N_{r}=12$. Cette rupture est telle que l'estimation des paramètres AR avant la rupture consuit à l'estimation de $\underline{a}$ et celle après la rupture donne le vecteur $\underline{c}$ (identique au vecteur paramètre estimé sur la somme des deux $A R$ considérés précédemment). Le "pic" n'apparait plus.

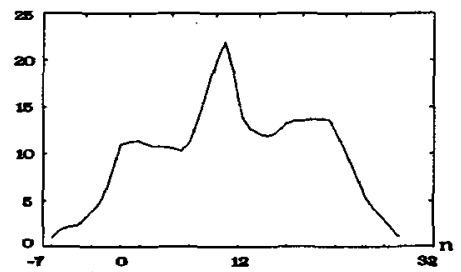

fig 3 : Détection de la forme en l'absence de bruit

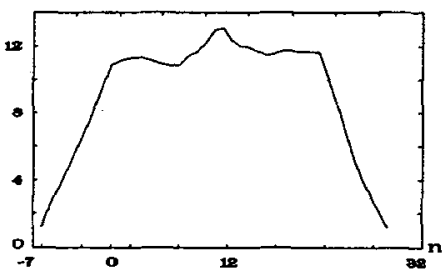

fig 4 : Détection de la forme recherchée dans un signal bruité $S N R=0.5 d b$

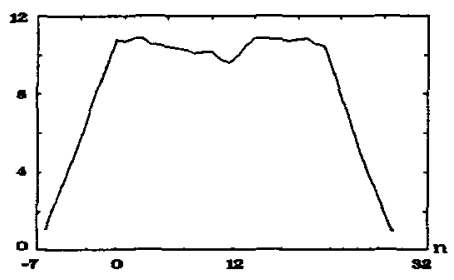

fig 5 : Détection de la forme recherchée dans un signal bruité

Nous voyons donc sur les figures ci-dessus que l'on peut reconnaître la présence d'une rupture additive et déterminer l'instant de rupture, cela même en présence de bruit.

\section{Conclusions}

Dans l'analyse de signaux non-stationnaires, présentant une rupture brutale additive, la modélisation AR glissante (sans détection préalable de l'instant de rupture) conduit à des coefficients dépendant du temps. Dans cet article, nous avons déterminé l'expression théorique de ces coefficients AR à chaque instant. Nous avons vérifié par simulations la bonne adéquation des résultats théoriques et des résultats expérimentaux. L'expression théorique de ces coefficients permet, par une méthode basée sur un calcul de corrélation, de détecter la présence d'une rupture additive sur un signal ainsi que l'instant de cette rupture.

\section{Références}

[1] S.KAY "Modern Spectral Estimation : Theory and Application",Prentice Hall 1988.

[2] A.ROSENFELD \& A.C.KAK "Digital picture Processing",Academic Press 1976 